\title{
Investigating the Influence of Colors on the Performance of Pointing Tasks for Human Interface Design
}

\author{
Jing KONG ${ }^{\dagger \mathrm{a})}$, Nonmember, Xiangshi REN ${ }^{\dagger \dagger}$, and Keizo SHINOMORI ${ }^{\dagger \dagger}$, Members $^{\circ}$
}

SUMMARY Fitts' law has been applied in many studies to evaluate pointing tasks. However, the quantitative effect of using color in the interfaces has not been discussed in the literature. This paper introduces research on the effects of color in pointing tasks using Fitts' law as the evaluation method. Different colors and color presentation styles are applied in the experiments which are similar in design to the paradigmatic Fitts' law pointing task. The experimental results show that when the subjects use a mouse as the input device, there is no significant difference between an interface with a colored target and one with a white target in the mean performance time. The results also reveal that color presentation styles will offer no significant difference to pointing tasks when the mouse is applied. However, when the interface of tablet PC and pen was applied, subjects without much experience in tablet personal computer usage needed more time to perform the task with colored targets than with a white target. Furthermore, when the colors are changed randomly during the selection process, the difference is even more obvious. These results are confirmed by a Checking Experiment and a Learning Effect Experiment which we performed on different groups of subjects.

key words: pointing, Fitts' law, modeling, human performance, color

\section{Introduction}

The pointing task is a ubiquitous performance in Human computer interaction (HCI). With the tremendous speed at which the technology is developing, numerous new pointing interfaces have appeared on the market, such as mouse, tablet pen, finger, trackball, eye-tracker, etc. Each of these devices has its own features and correspondingly different niche in HCI applications. Therefore, we need to use models to give clear evaluations of the different devices. For pointing tasks, the most famous model for evaluation and prediction is Fitts' law, which was proposed in 1954 by Paul Fitts [4], and has been widely used to predict and evaluate the performance of rapid and aimed movements. Now the most popular format of Fitts' law is as follows [8]:

$$
M T=a+b \log _{2}\left(\frac{A}{W}+1\right)
$$

where $a$ and $b$ are constants, $A$ is pointing distance, $W$ is the target width that limits the pointing accuracy tolerance demanded by the task, and MT is the expected average movement time of task completion.

\footnotetext{
Manuscript received October 14, 2005.

Manuscript revised April 4, 2006.

${ }^{\dagger}$ The author is with Nagoya University, Nagoya-shi, 464-8601 Japan, and Harbin Engineering University, P.R. China.

${ }^{\dagger}$ The authors are with Kochi University of Technology, Kamishi, 782-8501 Japan.

a) E-mail: Kong@watanabe.ss.is.nagoya-u.ac.jp

DOI: 10.1093/ietisy/e90-d.2.500
}

The second term of Fitts' law is called the index of difficulty $(I D)[8],[10]$ :

$$
I D=\log _{2}\left(\frac{A+W}{W}\right)
$$

We call Eq. 2 the $I D$ model.

However, according to Fitts, during the task, the hits made by the subjects need to follow a normal distribution. Usually when the researchers apply this model, they assume that the distribution of hits follows the normal distribution accurately and therefore it is not necessary to consider the difference in performance accuracy in the pointing task. This is not always the reality [5], [14], [15].

A model utilizing effective target width $\left(W_{e}\right)$ instead of the appointed target width $(W)$ also has the support of researchers [2], [10], [13]. $W_{e}$ indicates the actual input hits range based on performers' behavior during the task.

$$
M T=a+b \log _{2}\left(\frac{A}{W_{e}}+1\right)
$$

Simultaneously, one may calculate the effective index of difficulty $\left(I D_{e}\right)$ as:

$$
I D_{e}=\log _{2}\left(\frac{A}{W_{e}}+1\right)
$$

where $W_{e}=4.133 \sigma$.

$\sigma$ is the standard deviation of the hits distribution. In this paper we call this version of Fitts' law the $I D_{e}$ model. It has been accepted by ISO standards 9241-9 [6]. ISO standards 9241-9 proposed to use Throughput to evaluate the performance efficiency of pointing tasks.

$$
\text { Throughput }=\frac{I D_{e}}{M T}
$$

By using $W_{e}$, the $I D_{e}$ model reconciles the performance accuracy into the movement time to evaluate the performance and make the model more reasonable. However, neither the $I D$ nor the $I D_{e}$ models are based on mathematical deduction, but they have gained support respectively [4], [13]. Therefore, we regard that both models are applicable for one-dimensional pointing task evaluation, only with different application advantages.

Fitts' law (both the $I D$ and $I D_{e}$ model) has been applied widely in human computer interaction since 1978 even with a lack of theoretic support [1], [11]. The $I D_{e}$ model has even been accepted by ISO standards 9241-9 [6]. However, 
although most of the displays in human computer interaction include color, all the existing researches of the pointing task related to Fitts' law, both theoretic and applied, are based on black and white interfaces. The effects of color on motor tasks related to Fitts' Law have not been considered. Therefore, the purpose of this paper is to use Fitts' law (the $I D$ and $I D_{e}$ model) to evaluate the effect of color on pointing tasks. This will be a new horizon for Fitts' law applications.

Although there have been studies about colored stimuli, some of them concentrate on the role of color in the perception of motion in animated visualization in the field of Computer Graphics (CG) [12], while others concentrate on visual mechanisms with colored stimuli [3]. However, the color's direct effects on performance have not been studied sufficiently. Therefore, the results of this study will be useful for research and interface development in the HCI field.

Since the purpose of this paper is to test the color's comprehensive effect on human computer interaction performance through Fitts' law models, we included the effects of luminance and saturation changes together in the color's effects by applying the primary colors (red, blue and green) defined by JAVA environment on different apparatus. By this way, we can examine the effect of color on the users' performance in the usual cases.

\section{Experiment 1: On Interface with Fixed Colors}

First we measured the effect of color in a pointing task when the colors of the targets were fixed.

\subsection{Subjects}

Eleven volunteers, of different genders and ages (20 to 29 years, nine males and two females, average 22.3 years old) participated in a pointing experiment. All the subjects were right hand dominant.

\subsection{Apparatus}

We used two sets of apparatus to test the influence of color on human performance in HCI interfaces. The first set of apparatus included a Tablet Computer (FUJITSU FMV Stylistic, with a screen size of $21 \mathrm{~cm} \times 15.6 \mathrm{~cm}$, each pixel on the screen was $0.2055 \mathrm{~mm}$ wide) and a plastic pen. The second set of apparatus included a desktop personal computer (screen size: $43 \mathrm{~cm} / 17.0$ Diagonal, pixel pitch: $0.264 \mathrm{mmH} \times 0.263 \mathrm{mmV}$, each pixel on the screen was $0.264 \mathrm{~mm}$ wide) and a mouse (Agiler AGM 6124X).

\subsection{Procedures}

Similar to the original Fitts experiment [4], participants did reciprocal pointing on a pair of vertical strip targets with the plastic pen or the mouse according to the experimenters' instructions. The widths $(W)$ of the target were set at $W=$ $12,36,72$ pixels and the center to center distances or amplitudes $(A)$ between the two strips were set at $A=120,360$,

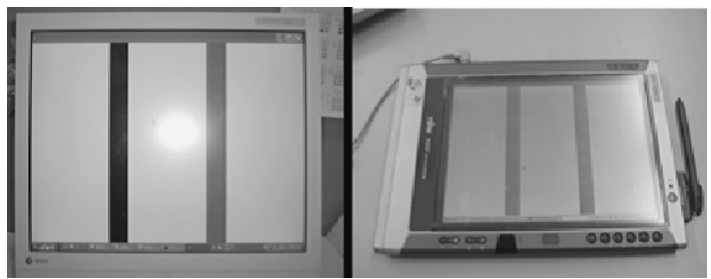

(a) Desk-top PC

(b) Table PC

Fig. 1 Experiment interfaces (when the target is blue).

Table 1 Error rates and accidental trials of Exp. 1.

\begin{tabular}{c|c|c}
\hline Apparatus and colors & Error rates & Accidental trials \\
\hline Pen (mix) & 0.016 & 5 \\
\hline Pen (red) & 0.017 & 0 \\
\hline Pen (green) & 0.019 & 2 \\
\hline Pen (blue) & 0.013 & 3 \\
\hline Mouse (mix) & 0.024 & 5 \\
\hline Mouse (red) & 0.033 & 1 \\
\hline Mouse (green) & 0.023 & 3 \\
\hline Mouse (blue) & 0.018 & 1 \\
\hline
\end{tabular}

840 pixels. The consequent $I D$ s of different $A-W$ combinations were decided by Eq. 2 . The order of the $9 A-W$ combinations was randomized. 12 trials were presented for each pair of targets, with the first tap excluded in the analysis. If the subject tapped outside of the target, an auditory signal was played.

During the task, the non-target rectangle was black, while the color of the target rectangle changed from the regularly used white into one of the primary colors (red, blue and green) in one $A-W$ combination. Once tapped, the position of the target rectangle and the non-target rectangle were reversed. The appearance of the three colors was balanced by a Latin square sequence and set by the experimenter before the subject began to tap. Therefore the total number of trials afforded for one subject to fulfill was 3 (colors) $\times 3$ (distances) $\times 3$ (widths) $\times 12$ (trials) $=324$.

The interface of the experimental tool is shown in Fig. 1 and the details about the target colors are described in Appendix A.

\subsection{Error Rates}

During the experiment, if the subject tapped on the region outside the target, an auditory signal would be played, and simultaneously, a mistake would be recorded for future error rate calculation.

In fact, some participants sometimes clicked when their cursor was no where near the target due to the confusion of the participant. For example, sometimes the target appeared in the left part of the screen, but the subject accidentally pointed to the right part of the screen. Usually in HCI, we delete these data in analysis, because there are no necessary relationships between the occurrence of the accidental trials and the factors that we want to study [9], [15]. The information regarding the accidental trials and error rates in Experiment 1 is listed in Table 1. 


\section{Experiment 2: On Interface with Randomly Chang- ing Colors}

Through Experiment 1, we were able to examine the effects of colors on pointing performance, but we also wanted to study the effects of different color presentation styles on task performance. Therefore, we carried out Experiment 2 asking the subjects to perform the pointing task in an interface with randomly changing colors. The expression "randomly changing colors" means that the colors of the target rectangle were changed randomly using the three appointed colors (red, green and blue) as described in Fig. A. 1 during one section of the $A$ and $W$ combination, i.e. while doing the pointing task, the color of the target changed randomly without any warning after each pointing.

\subsection{Subjects}

The same subjects in Experiment 1 also took part in Experiment 2.

\subsection{Apparatus}

We used the same apparatus in Experiment 2 as in Experiment 1 .

\subsection{Procedures}

The procedure of the task was the same as that in Experiment 1 . The total number of the trials afforded for one subject to fulfill was 3 (distances) $\times 3$ (widths) $\times 12$ (trials) $=$ 108.

\subsection{Error Rates}

We also recorded the mistakes and deleted accidental trials from the data as described in Sect. 2.4. The error rates and accidental trials of the subjects with different apparatus and interfaces are shown in Table 2.

\section{Non-color Experiment}

To make a comparison of the effects between white and colored targets in the pointing task, we utilized some data from the experiments we had developed previously [7], [15] and

Table 2 Error rates and accidental trials of Exp. 2.

\begin{tabular}{c|c|c}
\hline Apparatus and colors & Error rates & Accidental trials \\
\hline Pen (mix) & 0.019 & 18 \\
\hline Pen (red) & 0.012 & 4 \\
\hline Pen (green) & 0.027 & 7 \\
\hline Pen (blue) & 0.018 & 7 \\
\hline Mouse (mix) & 0.027 & 1 \\
\hline Mouse (red) & 0.022 & 0 \\
\hline Mouse (green) & 0.027 & 0 \\
\hline Mouse (blue) & 0.032 & 1 \\
\hline
\end{tabular}

called the related experiment Non-color experiment.

One part of the Non-color Experiment using a tablet personnel computer and pen was developed by [15], where the two models, $I D$ and $I D_{e}$, have both been discussed thoroughly. Fifteen volunteers, 5 female and 10 male, aged 20 to 36 years old, participated in that project. For comparison, we picked up part of the data of the experiment conducted by Zhai et al. [15] which had a similar procedure to Experiment 1 . During this part of the experiment, the target rectangle was always white and the non-target rectangle was always black. The error rate for the task was $4 \%$, and one accidental trial was excluded from the data for analysis.

The other part of the Non-color Experiment using a regular personnel computer and mouse was developed by [7]. Twelve subjects, of different genders and ages ( 3 female students and 9 male students, 21 to 32 years old, average age 25) participated in the experiment. All subjects were right hand dominant. The procedure of this part of that experiment was similar to our Experiment 1. During that part of the experiment [7], the target rectangle was always white and the non-target rectangle was always black. The error rate of the task was $2 \%$, and no accidental trials occurred during the experiment.

\section{Checking Experiment}

Because the subjects for the experiments with colored targets were different to those we used in the experiments with the white targets, we asked 5 subjects to perform all the experiments mentioned above so as to make the comparison reliable, (Exp. 1, Exp. 2 and Non-color Exp.) and we organized the data calling it Checking Experiment ${ }^{\dagger}$. However, in the Checking Experiment, since our purpose was merely to test the reliability of the comparison results of Exp. 1, Exp. 2 and the Non-color Exp., we asked the subjects to perform the pointing task only under one $A-W$ combination ( $A=840$ pixels, $W=12$ pixels). The reason for this choice was that with a low level of difficulty, different colors may not incur much difference in performance, but with a bigger task difficulty, the difference can be significant.

\section{Results and Discussions}

Since both the $I D$ model and $I D_{e}$ model of Fitts' law have obtained support, we show the comparison results by applying both models.

\subsection{Difference Incurred by Different Colors}

First, we performed ANOVA and found that there was no significant statistical difference among the mean times of the different colors afforded by the experiment if we used the

\footnotetext{
${ }^{\dagger}$ The purpose of the Checking Exp. was to check whether the comparison results of the experiments with different subjects are identical. The subjects included in the Checking experiment needed to perform all the experiments that had been performed by different sets of subjects.
} 


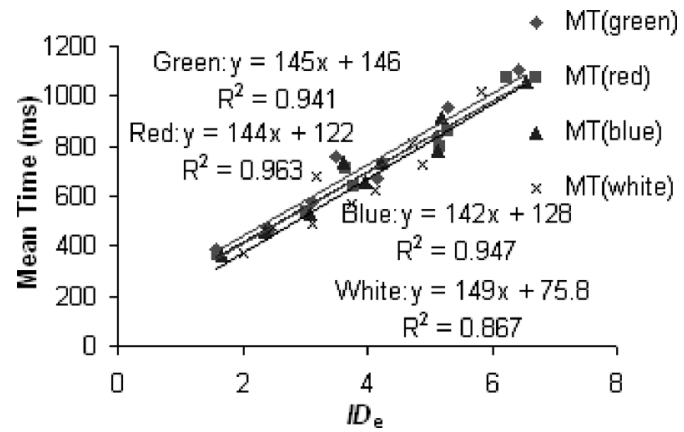

Fig. $2 M T-I D_{e}$ regression lines with different colors of the target in Exp. 1 and Non-color Exp. (pen).

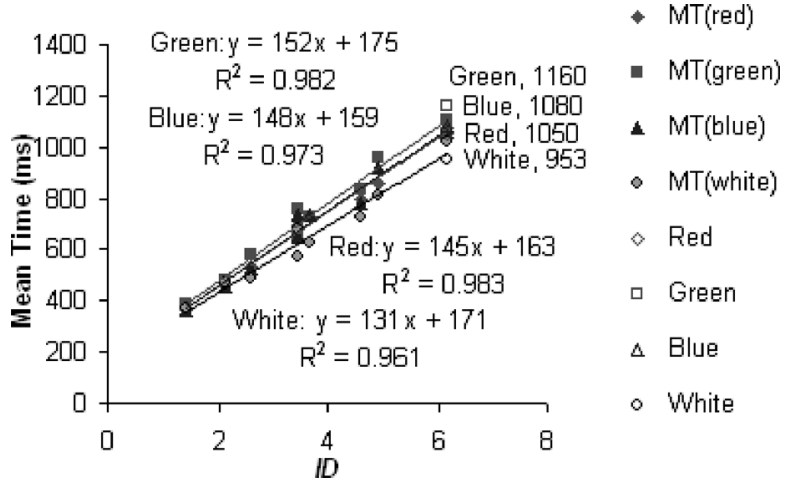

Fig. $3 \quad M T-I D$ regression lines with different colored targets in Exp. 1 and the Non-color Exp. (pen) and the $M T-I D$ relationship with different colored targets in Checking Exp. of Exp. 1 and the Non-color Exp. (pen) (The open symbols denote the data points of the Checking Experiment).

pen as the input device.

However, from the Fitts' law analysis (based on $I D$ and $I D_{e}$ ), we can still observe that the colored targets consumed more time on average than the white targets (Figs. 2 and 3).

Figure 3 also shows the Checking Experiment results of Exp. 1, through which we were able to test whether the sequences of the time cost pertaining to different colors for different groups of subjects were reliable. We did a T-test to test the statistical significance of the difference between different colors in the Checking Exp. There is a significant difference between white and red $\left(P_{108}(t \geq 2.23)<0.05\right)$, white and green $\left(P_{108}(t \geq 5.13)<0.0001\right)$, white and blue $\left(P_{108}(t \geq 3.49)<0.001\right)$, green and blue $\left(P_{108}(t \geq 2.29)<\right.$ $0.05)$, red and green $\left(P_{108}(t \geq 2.48)<0.05\right)$, there is no significant difference between blue and red.

Therefore, the results of the Checking Exp. supported the comparison sequences of Exp. 1 and of the Non-color Exp.: i.e. colored targets consume more time than the white target. Nevertheless, the difference between the performance with different colors (red, green and blue) is not significant. Meanwhile, Table 1 and the results of error rates from the Non-color Exp. show that during the task with different colors, the subjects made fewer errors with colors compared to the one without color ${ }^{\dagger}$.

ANOVA shows that there was no significant statistical

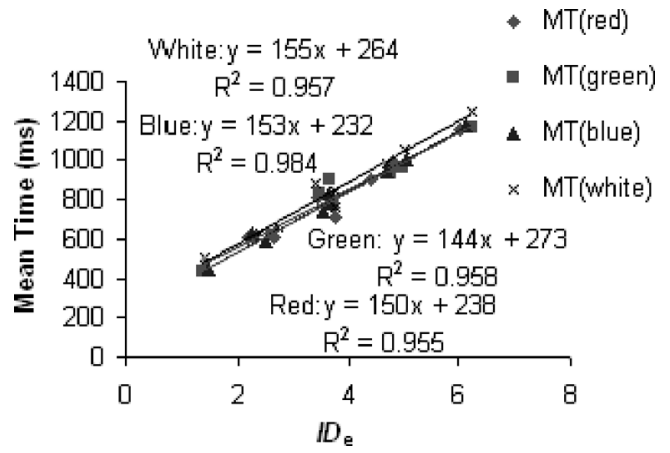

Fig. $4 \quad M T-I D_{e}$ regression lines with different colored targets in Exp. 1 and the Non-color Exp. (Mouse).

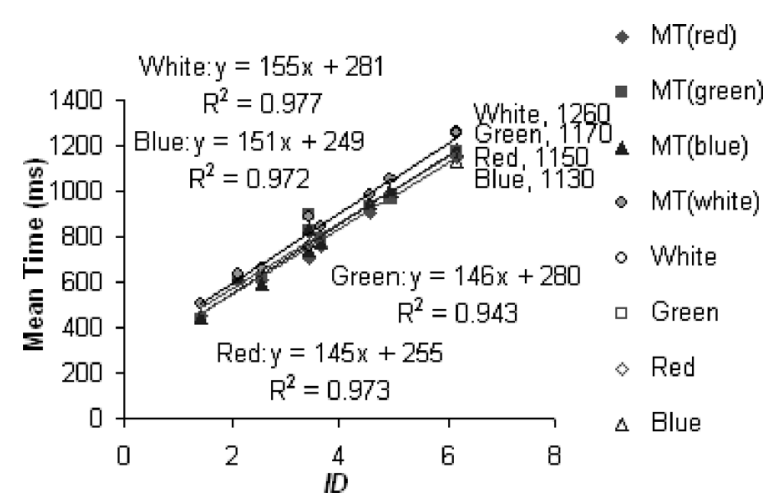

Fig.5 $M T-I D$ regression lines with different colored targets in Exp. 1 and the Non-color Exp. (Mouse) and the $M T-I D$ relationship with different colored targets in the Checking Exp. Of Exp. 1 and the Non-color Exp. (Mouse).

difference among the mean times for the different colors afforded by the experiment if we used the mouse as the input device.

Fitts' law regression lines (Figs. 4 and 5) also show that using a mouse and a desk-top PC, targets in different colors affect almost no difference in mean time even when we vary the difficulty of the task.

T-test results of the Checking Exp. only show a significant difference between white and red $\left(P_{108}(t \geq 2.19)<\right.$ $0.05)$, white and blue $\left(P_{108}(t \geq 2.61)<0.05\right)$, but not between blue and red, white and green, green and blue, red and green.

Therefore, after the Checking Exp., even though there is a tiny difference between the regression lines in Figs. 4 and 5 and the T-test analysis, we think that the difference caused by different colored targets can be ignored when the mouse is used as the input device.

\subsection{Differences Incurred from the Color Presentation Styles}

Next we tested the difference caused by the color presentation styles.

\footnotetext{
${ }^{\dagger}$ This difference of error rates is included in the $I D_{e}$ Model.
} 


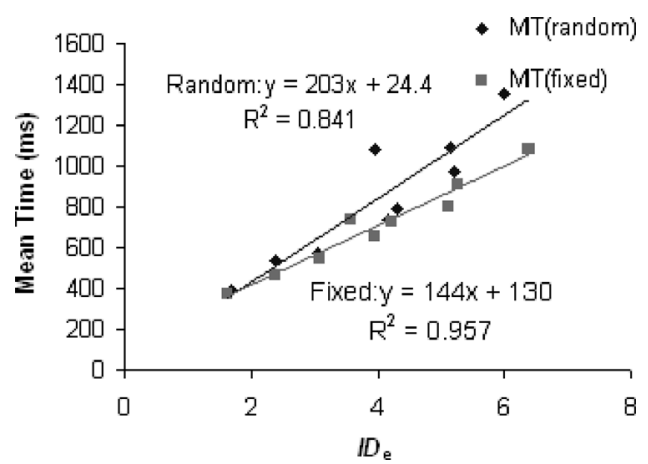

Fig. $6 M T-I D_{e}$ regression lines of the mixed data of three colors in Exp. 1 (fixed) and Exp. 2 (random). (pen).

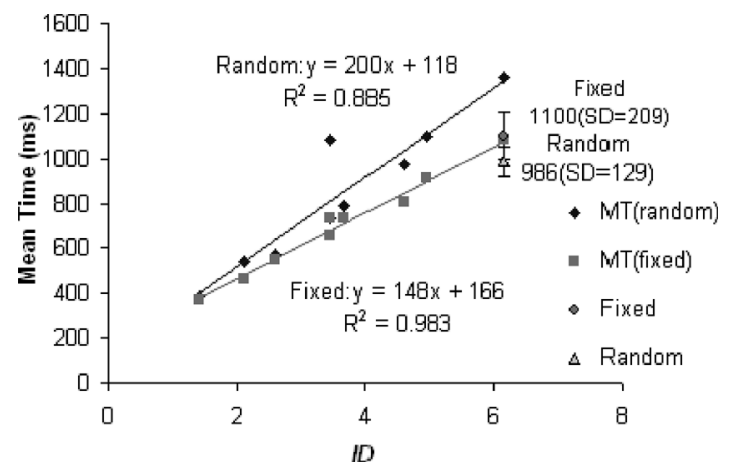

Fig. $7 M T-I D$ regression lines of the mixed data for three colors in Exp. 1 (fixed) and Exp. 2 (random) (pen) and the $M T-I D$ relationship of the mixed data for three colors in the Checking Exp. Of Exp. 1 (fixed) and Exp. 2 (random). (pen).

\subsubsection{Analysis of the Data with Pen}

According to ANOVA, there is no significant statistical difference among the mean times for different color presentation styles with the pen as the input device.

However, Figs. 6 and 7 coherently show that the targets displayed in randomly changing colors consumed more performance time than the targets displayed in a fixed color. When the difficulty increased, subjects needed more time to track the targets which were displayed in randomly changing colors. The difference in the data regarding performance time with the pen is obvious.

However, the Checking Exp. results contradict the comparison results of Exp. 1 and 2 (see Fig. 7).

We did a T-test to test the statistical significance of the difference in mean performance time between different color presentation styles of the Checking Exp. There is significant difference between the data from the task with randomly changing colors and the data from fixed color tasks $\left(P_{218}(t \geq 3.77)<0.001\right)$.

One potential reason for the conflicts between the Checking Exp. and Exp. 1 and 2 may be the different pointing task participation experience of the subjects in Exp. 1 and 2 and the subjects in the Checking Exp. In both Exp. 1 and 2, five of the total 11 subjects had some previous expe-

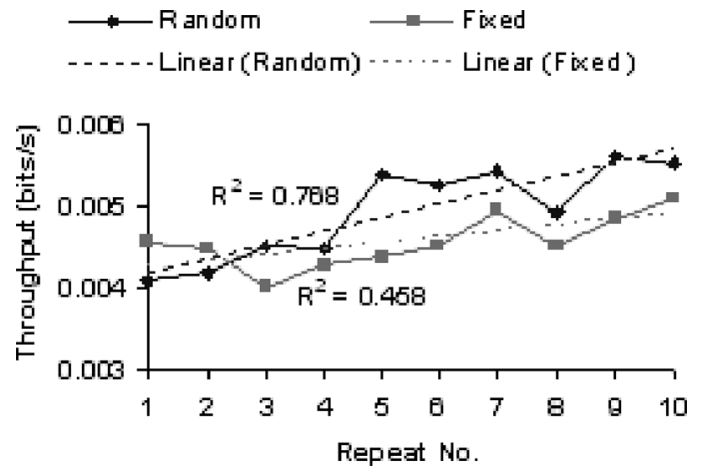

Fig. 8 Learning Effect Experiment results in throughput with the randomly changing color task of Group A and the fixed color task of Group B from Day 1.

rience using the tablet PC and had also taken part in similar pointing tasks executed in the lab previously. The other participants were completely new to tablet $\mathrm{PC}$ performance. On the other hand, all five subjects in the Checking Exp. had more than one hour experience with similar pointing tasks, therefore, the conflicts between the Checking Exp. and Exp. 1 and 2 may imply some learning effects of the pointing task.

Therefore, we carried out an experiment to check the learning effects.

\section{Learning Effect Experiment}

Eight subjects without either pointing task experience or experience using a tablet PC were involved in the Learning Effect Experiment. Each of the subjects took part in the experiment in 20 sessions over two successive days (20 repeats). The subjects were divided into two groups with four persons in each group. The subjects in Group A performed 10 repeats of the randomly changing colors task on the first day, and 10 repeats of the fixed color task on the second day. For the subjects in Group B, the sequence of the random color task and fixed color task was reversed. The break between repeats was 1 hour. During each session, the task procedure was similar to that in the Checking Exp. except that in the randomly changing color experiment part, the subjects performed 6 trials three times with one repeat, and in the fixed color experiment part, the subjects performed 6 trials for each color with one repeat. The total number of trials for each subject was $360=6 \times 3 \times 20$. Altogether 21 misses were exempted from analysis.

We checked the learning effect based on Throughput (Eq. 5), which is decided by considering both performance times and error rates according to ISO9241-9 standard [6].

The result shown in Fig. 8 shows that the throughput was increased with practice for both tasks, and for the randomly changing color task, the improvement was greater.

Through the data of Day 2, we know that after the practice of Day 1, the performance in both tasks tends to be more stable. No more learning effects can be clearly observed through Fig. 9.

Thus the results of the Learning Effect Exp. make it easy to explain the conflict between the Checking Exp. and 


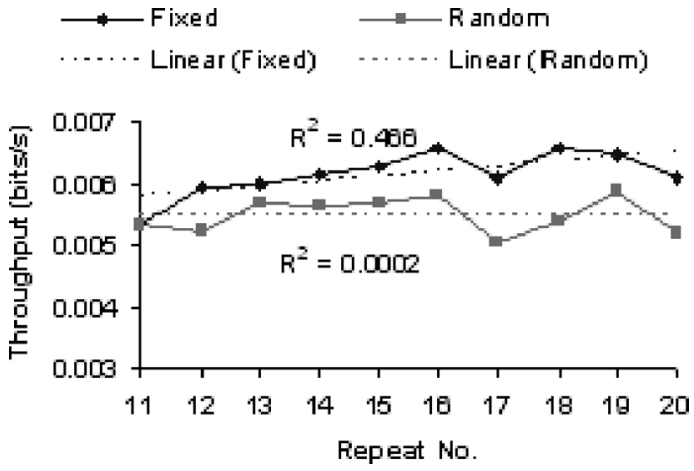

Fig. 9 Throughput for the fixed color task of Group A and the randomly changing color task of Group B from Day 2.

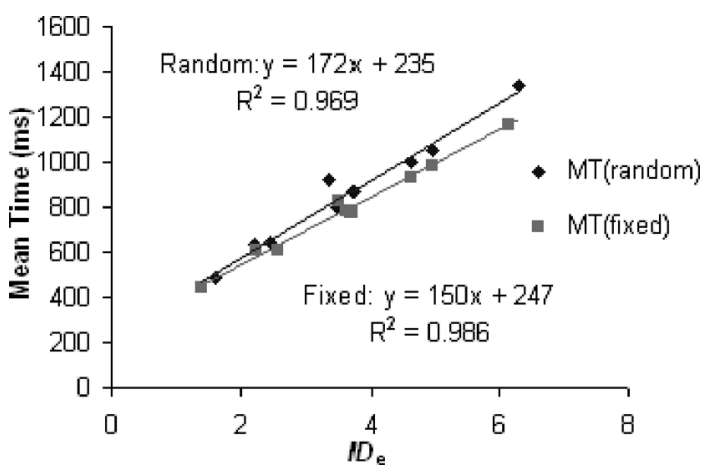

Fig. $10 M T-I D_{e}$ regression lines of the mixed data for three colors in Exp. 1 (fixed) and Exp. 2 (random). (Mouse).

Exp. 1 and Exp. 2. Since in the Checking Exp., all the subjects were experienced in pointing tasks and tablet PC usage, their performances for the two kinds of tasks were closely matched. On the other hand, in Exp. 1 and Exp. 2, the novice subjects needed more time to fulfill the randomly changing color task than the fixed color task. After training, it will cost similar time for the subjects to perform the randomly changing color task and the fixed color task.

\subsubsection{Analysis of the Data with Mouse}

According to ANOVA, there is no significant statistical difference among the mean times for different color presentation styles with mouse as the input device.

When the mouse was used as the input device, the Checking Exp. results were identical to those obtained from Exp. 1 and 2, and the time difference for performing with Random Color and Fixed Color tasks is shorter than that for using the pen as the input device (see Fig. 10 and Fig. 11). This could be because the mouse is a familiar tool for all the subjects (T-test results show that there is no significant difference between the randomly changing colors task and fixed color tasks). Even though some of the subjects had no experience using a mouse to perform the pointing task designed in this paper, their abundant experience using a mouse in general computing helped them adapt to the task quite easily.

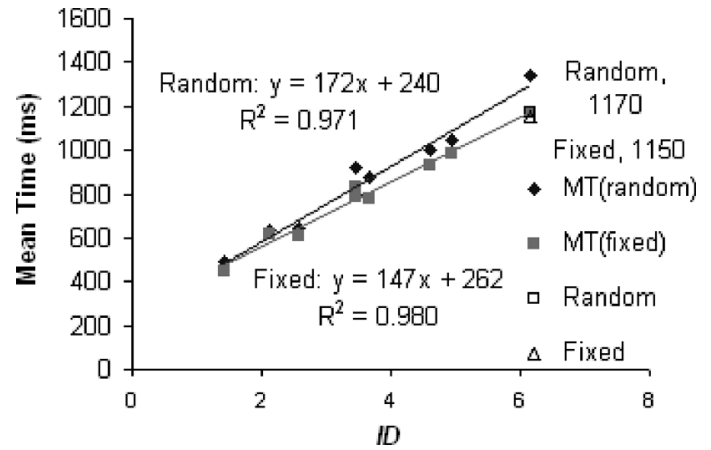

Fig. $11 M T-I D$ regression lines of the mixed data for three colors in Exp. 1 (fixed) and Exp. 2 (random) (Mouse) and the $M T-I D$ relationship of the mixed data for three colors in the Checking Exp. of Exp. 1 (fixed) and Exp. 2 (random). (Mouse).

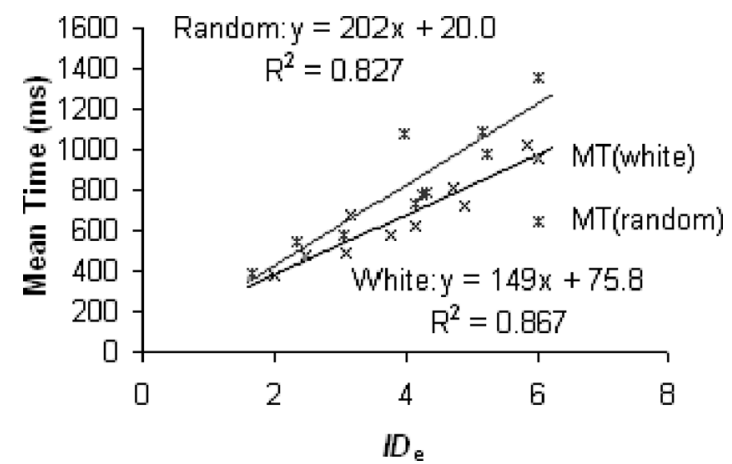

Fig. $12 M T-I D_{e}$ regression lines of the interface with color (Exp. 2) and without color (Non-color Exp.) (pen).

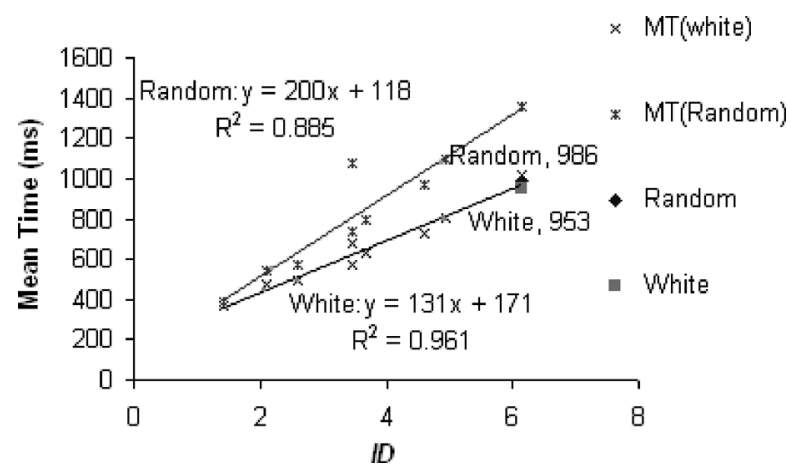

Fig. $13 M T-I D$ regression lines of the interface with color (Exp. 2) and without color (Non-color Exp.) (pen) and the $M T-I D$ relationship of the checking Experiment of the interface with color (Exp. 2) and without color (Non-color Exp.) (pen).

\subsection{Differences Incurred by the Colored Interfaces}

Finally we compared the results of the experiments with and without colors.

The ANOVA results show that there is no significant difference among the mean times for the colored interfaces and the black and white interfaces, either with fixed colors or randomly changing colors, pen or mouse. 


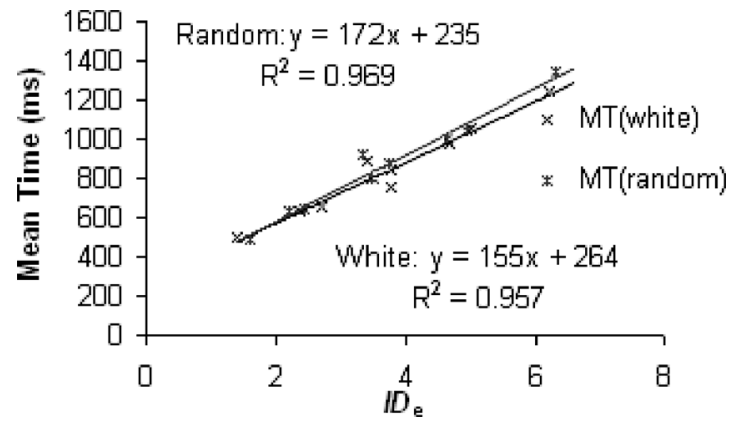

Fig. $14 M T-I D_{e}$ regression lines of the interfaces with color (Exp. 2) and without color (Non-color Exp.) (Mouse).

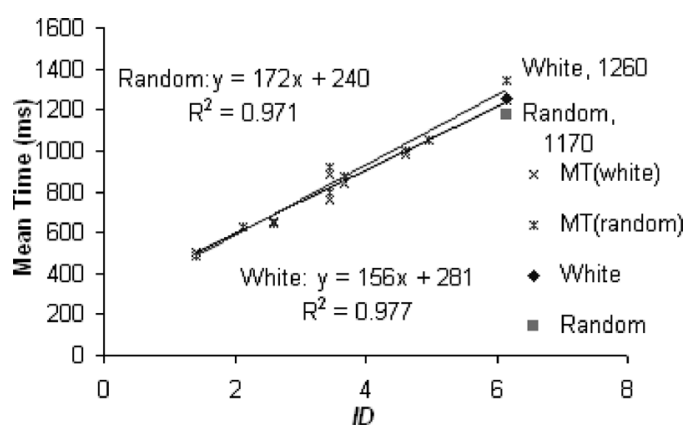

Fig. $15 M T-I D$ regression lines of the interfaces with color (Exp. 2) and without color (Non-color Exp.) (Mouse) and the $M T-I D$ relationship of the Checking Exp. of the interfaces with color (Exp. 2) and without color (Non-color Exp.) (Mouse).

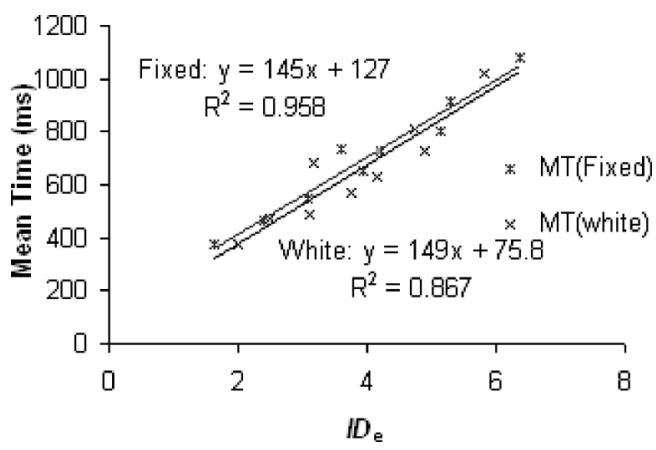

Fig. $16 M T-I D_{e}$ regression lines for the interface with color (Exp. 1) and without color (Non-color Exp.) (pen).

Nevertheless, Fig. 12 and Fig. 13 show that with the randomly changing colors in the interface, subjects need more time to track the target when using a pen.

The results of the Checking Exp. are identical to the comparison results (Fig. 13). T-test results show that there is no significant difference between the fixed color and randomly changing color tasks of the Checking Exp.

However, the difference in movement time is not clear when a mouse was applied as the input device (see Fig. 14 and Fig. 15).

The comparison results of the Checking Exp. are not the same as the comparison between Exp. 2 and the Noncolor Exp. Nevertheless, the T-test shows no significant dif-

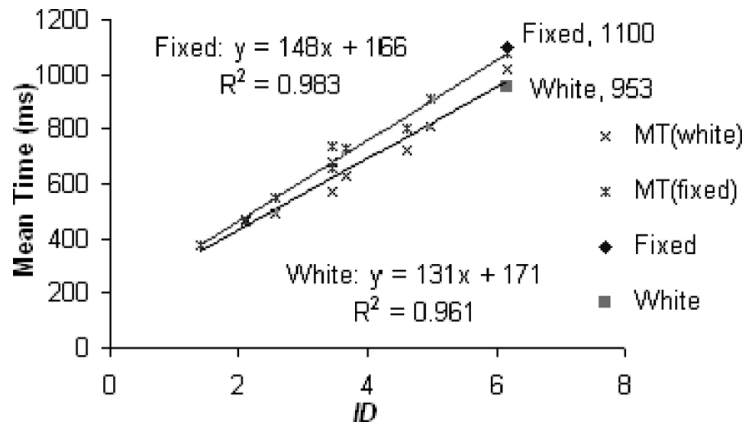

Fig. $17 M T-I D$ regression lines for the interfaces with color (Exp. 1) and without color (Non-color Exp.) (pen) and the $M T-I D$ relationship of the Checking Exp. of the interfaces with color (Exp. 1) and without color (Non-color Exp.) (pen)

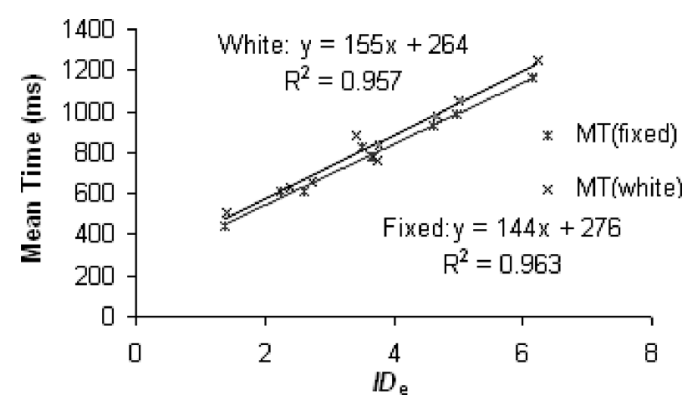

Fig. $18 M T-I D_{e}$ regression lines for the interfaces with color (Exp. 1) and without color (Non-color Exp.) (Mouse).

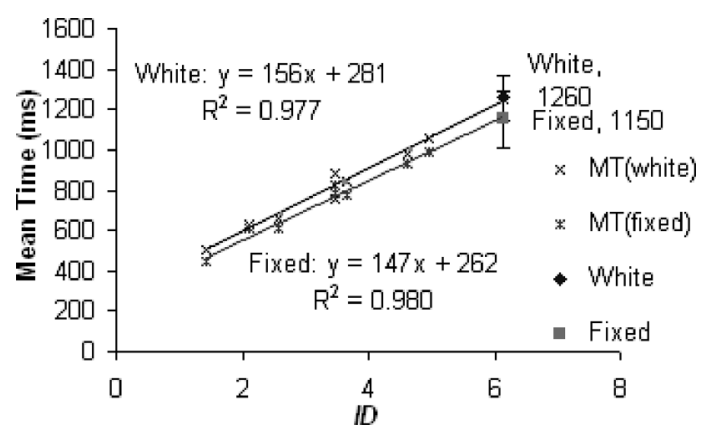

Fig. $19 M T-I D$ regression lines of the interfaces with color (Exp. 1) and without color (Non-color Exp.) (Mouse) and the $M T-I D$ relationship of the Checking Exp. of the interfaces with color (Exp. 1) and without color (Non-color Exp.) (Mouse).

ference exists between the Non-color and randomly changing color styles, therefore, we may ignore the discrepancy.

When the colors of the target are fixed, there is no obvious difference among the colored interfaces and the noncolor interface either with pen or mouse (see Fig. 16, Fig. 17, Fig. 18 and Fig. 19). Although the comparison results made by Exp. 1, Non-color Exp. and the Checking Exp. are not the same, since in either group of comparison there is no significant statistical difference and the difference of direct observation of the regression lines is also tiny, the difference between the non-color tasks and the fixed color task can be ignored. 


\section{Conclusions}

In this paper, we used Fitts' law and ANOVA to thoroughly compare the effects of using colors in pointing tasks. Three colors (red, green and blue) were applied in the experiments. Moreover, we also tested the effect of different color presentation styles during the pointing task (fixed colors and randomly changing colors). In case there could be discrepancies caused by different subjects in the color and non-color experiments, we also carried out a Checking experiment for the three experiments (Exp. 1, Exp. 2 and Non-color Exp.). A Learning Effect Experiment was executed to explain the relationship between the Checking Exp. and the main experiments.

The great regression of the relationship of mean time and $I D$ or $I D_{e}$ demonstrates that Fitts' law is effective for device evaluation of interfaces with colored targets.

With the experimental data, it is not difficult to make conclusions on the effects of colors:

1. in pointing tasks, different colors will not produce significant differences in performance. However, when the subjects use a pen, they need more time to perform the color task.

2. in the tasks with randomly changing colors, the performance of the novice subjects is worse than in the task with a fixed color. However, the difference is insignificant when the subjects use the mouse.

3 . in the task with colors (red, green and blue) and without color (white), if the color is fixed when the target is tapped, performance remains almost constant.

For the effects of different colors and different input devices, we can conclude that the performance situation will be different, but the differences are not big. When people use a pen to tap the target with randomly changing colors, the performance time is a little longer than when they tap the target without color or without changing color. Using the pen, there is no friction to limit the speed of the pen, and sometimes it is difficult to change the accelerating power so as to adjust the route of the pen. If the target's color is changed without warning, it is difficult for the subjects to make changes according to their visual feedback. However, these phenomena were only apparent for the novice subjects. For the experienced subjects, the difference is reduced. When people use the mouse, there is friction, so the speed is not very fast and it is easier for subjects to adjust the performance power when the mouse approaches the target, regardless of whether the target is white, colorful or even in randomly changing colors.

These conclusions imply that even though there is no big difference for different colored targets in normal circumstances, in some special situations, for instance, for a novice user, or when using a tablet pen, designers need to deliberatively consider the application of colors and color presentation styles.

\section{References}

[1] S.K. Card, W.K. English, and B.J. Burr, "Evaluation of mouse, rate controlled isometric joystick, step keys and text keys for text selection on a CRT," Ergonomics, 21, pp.601-613, 1978.

[2] E.R.F.W. Crossman and P.J. Goodeve, "Feedback control of handmovement and Fitts' law," Quarterly J. Experimental Psychology, vol.35A, pp.251-278, 1983.

[3] R.F. Dougherty and W.A. Press, "Perceived speed of colored stimuli," Neuron, vol.24, pp.893-899, 1999.

[4] P.M. Fitts, "The information capacity of the human motor system in controlling the amplitude of movement," J. Experimental Psychology, vol.47, pp.381-391, 1954.

[5] P.M. Fitts and B.K. Radford, "Information capacity of discrete motor responses under different cognitive sets," J. Experimental Psychology, vol.71, no.4, pp.475-482, 1966.

[6] ISO, "ISO 9241-9 Ergonomic requirements for office work with visual display terminals (VDTs) - Part 9: Requirements for non-keyboard input devices (FDIS - Final Draft International Standard)," International Organization for Standardization, 2000. http://iso.w3j.com/index.html

[7] J. Kong, X. Ren, X. Jiang, and F. Takeda, "Comparison of four input devices for pointing task by applying SH-Model,” Proc. WEC'2004, pp.168-174, 2004.

[8] I.S. MacKenzie, "A note on the information-theoretic basis for Fitts' law," Journal of Motor Behavior, vol.21, pp.323-330, 1989.

[9] I.S. Mackenzie, A. Sellen, and W. Buxton, "A comparison of input devices in elemental pointing and dragging tasks," Proc. ACM Conference on Human Factors in Computing Systems - CHI '91, pp.161-166, 1991

[10] I.S. MacKenzie, "Fitts' law as a research and design tool in human computer interaction," Human-Computer Interaction, vol.7, pp.91139, 1992.

[11] I.S. MacKenzie and S.X. Zhang, "The design and evaluation of a high-performance soft keyboard," Proc. CHI 1999, pp.25-31, 1991.

[12] D. Weiskopf, "On the role of color in the perception of motion in animated visualizations," Proc. IEEE Visualization 2004, pp.305312, 2004.

[13] A.T. Welford, Fundamentals of Skill, Methuen, London, 1968.

[14] S. Zhai, "Characterizing computer input with Fitts' law parameters: The information and non-information aspects of pointing," Int. J. Human-Computer Studies, vol.61, no.6, pp.791-809, Dec. 2004.

[15] S. Zhai, J. Kong, and X. Ren, "Speed-accuracy trade-off in Fitts' law tasks: On the equivalency of actual and nominal pointing precision," Int. J. Human-Computer Studies, vol.61, no.6, pp.823-856, Dec. 2004

\section{Appendix: Measurement of Luminance and Color (Calibration)}

We measured luminance and chromatic coordinates of the colors that we used as stimuli by spectral radiometer (CS1000 made by Konica-Minolta). In measurement on tablet PC, we set the angle between the tablet PC and the detector of the radiometer in 66 degree from the horizontal surface, which was obtained as the average of 5 observers' experiments. Distance from the screen and the detector was $30 \mathrm{~cm}$ for the tablet PC and $50 \mathrm{~cm}$ for the PC (LCD), those were also obtained as the average of viewing distance of these observers.

Table A. 1 shows the luminance of the colors presented by the PC (LCD) and the tablet PC. This table shows that 
Table A. 1 Luminance and chromatic coordinates of stimuli used in PC (LCD) and tablet PC.

\begin{tabular}{|c|c|c|c|}
\hline Color of Stimuli & Values & SEM & Error rate \\
\hline Red & & & \\
\hline PC (LCD) Lv & 40.71 & +-0.78 & 1.92 \\
\hline u' & 0.4204 & +-0.0011 & 0.27 \\
\hline v' & 0.5271 & +-0.0007 & 0.14 \\
\hline Tablet PC Lv & 52.81 & +-1.09 & 2.06 \\
\hline u' & 0.2829 & +-0.0021 & 0.76 \\
\hline v' & 0.5144 & +-0.0011 & 0.22 \\
\hline Green & & & \\
\hline PC (LCD) Lv & 86.45 & +-1.78 & 2.06 \\
\hline u' & 0.1403 & +-0.0005 & 0.34 \\
\hline v' & 0.5632 & +-0.0013 & 0.23 \\
\hline Tablet PC Lv & 69.64 & +-1.12 & 1.61 \\
\hline u' & 0.1979 & +-0.0006 & 0.32 \\
\hline v' & 0.5272 & +-0.0038 & 0.72 \\
\hline Blue & & & \\
\hline PC (LCD) Lv & 16.94 & +-0.34 & 2.01 \\
\hline u' & 0.1295 & +-0.0012 & 0.90 \\
\hline v' & 0.2886 & +-0.0021 & 0.72 \\
\hline Tablet PC Lv & 44.98 & +-0.98 & 2.71 \\
\hline u' & 0.1996 & +-0.0035 & 1.76 \\
\hline$v^{\prime}$ & 0.4520 & +-0.0054 & 1.18 \\
\hline Background White & & & \\
\hline PC (LCD) Lv & 147.24 & +-2.47 & 1.68 \\
\hline u' & 0.2060 & +-0.0004 & 0.19 \\
\hline v' & 0.4816 & +-0.0018 & 0.37 \\
\hline Tablet PC Lv & 93.41 & +-0.41 & 0.44 \\
\hline u' & 0.2191 & +-0.0002 & 0.10 \\
\hline v' & 0.4940 & +-0.0004 & 0.07 \\
\hline
\end{tabular}

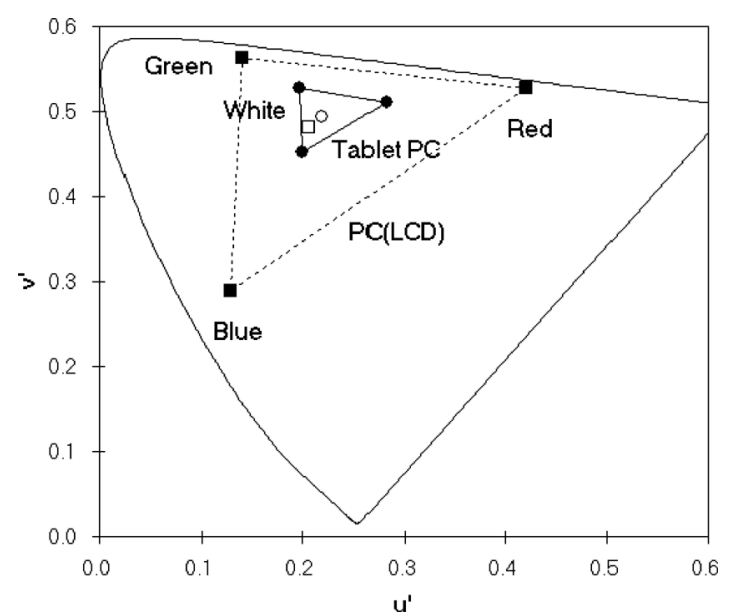

Fig. A. 1 CIE u'v' chromatic coordinates of stimulus colors in PC (LCD) and tablet PC. Squares and circles denote coordinates of colors by PC (LCD) and by tablet PC, respectively. Open symbols denote background white for each screen.

the luminance of the colors and the background white is different, therefore, we think that the target could be clearly perceived from the background white clearly.

Figure A. 1 shows the chromatic coordinates plotted in CIE u'v' coordinates. As shown in the figure, the colors presented by the tablet PC are closer to the background white compared to the ones by the PC (LCD). Although there is difference of saturation of the three colors (red, green and blue) in PC and tablet PC, we thought that since the aim of this research is to study the effect of colors on pointing performance, therefore, we should use the most saturated colors for each screen. Meanwhile, because the difference of the primary colors from white is large enough in both PC (LCD) and tablet PC, the difference of color saturation can be included in the changing stimulus' colors.

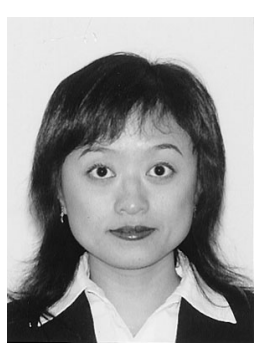

Jing Kong is a COE post-doctoral researcher in Nagoya University, Japan. She is also a staff member of Harbin Engineering University, China. She has received her bachelor's degree in communication engineering and master's degree in information and communication systems from Harbin Engineering University, China, in 1998 and 2003 respectively. She has obtained her Ph.D. in information systems engineering in Kochi University of Technology, Japan in 2006. Her current research interest includes human computer interaction modeling and cognitive support for creativity in knowledge handling. She is a member of ACM.

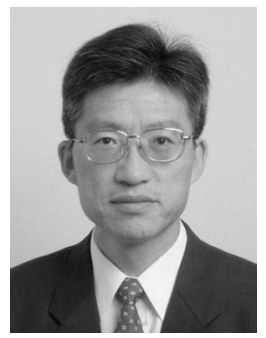

Xiangshi Ren is an associate professor in the Department of Information Systems Engineering at Kochi University of Technology. He received a B.E. degree in electrical and communication engineering, and M.E. and Ph.D. degrees in information and communication engineering from Tokyo Denki University, Japan, in 1991, 1993, and 1996, respectively. After working for Tokyo Denki University, he has been at Kochi University of Technology since 2000. His research interests include all aspects of humancomputer interaction, in particular, pen-based interactions, and user interface design and evaluation. He is a member of the IPSJ, and the Human Interface Society (all in Japan), the ACM, the ACM SIGCHI, the IEEE Computer Society, and the British HCI Group.

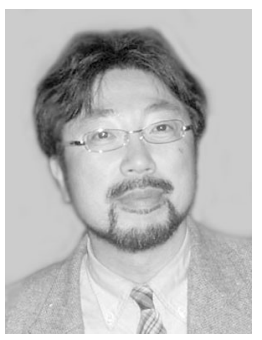

Keizo Shinomori is a professor in the Department of Information Systems Engineering and Frontier Engineering Course at Kochi University of Technology. He received B.S. degree from Science University of Tokyo in 1987. He received M.E. and Dr. Eng. from Tokyo Institute of Technology in 1989 and 1992, respectively. From 1992 to 1994, he was a Professional Research Associate of Department of Psychology, University of Colorado at Boulder, where he became a Graduate Faculty from 1995 to 1997 . He visited in University Freiburg in Germany as a Guest Scientist of Brain Research Group in Institute for Biophysics and Radiobiology from 1995 to 1996. Since 1997, he has been with the Faculty of Engineering, Kochi University of Technology. From 1997 to 1999, he was a visiting Associate Professor of Department of Psychology, University of Colorado at Boulder. Since 2000, he has been a visiting Professor of Department of Ophthalmology and Section of Neurobiology, Physiology, University of California, Davis. His main interest lies in the fields of vision psychophysics, color vision, age-related change in human vision, brain activities in vision and human-computer interaction. Dr. Shinomori is a member of VSJ (Vision Society of Japan), OSJ (the Optical Society of Japan), ARVO (the Association for Research in Vision and Ophthalmology), OSA (the Optical Society of America), ITE (the Institute of Image Information and Television Engineers), and IEIJ (the Illuminating Engineering Institute of Japan). 